\title{
Towards semen quality assessment using neural networks
}

\author{
Linneberg, Christian; Salamon, P.; Svarer, C.; Hansen, Lars Kai; Meyrowitsch, J.
}

Published in:

Proceedings of the 4th IEEE Workshop Neural Networks for Signal Processing

Link to article, DOI:

10.1109/NNSP.1994.366015

Publication date:

1994

Document Version

Publisher's PDF, also known as Version of record

Link back to DTU Orbit

Citation $(A P A)$ :

Linneberg, C., Salamon, P., Svarer, C., Hansen, L. K., \& Meyrowitsch, J. (1994). Towards semen quality assessment using neural networks. In Proceedings of the 4th IEEE Workshop Neural Networks for Signal Processing (pp. 509-517). IEEE. https://doi.org/10.1109/NNSP.1994.366015

\section{General rights}

Copyright and moral rights for the publications made accessible in the public portal are retained by the authors and/or other copyright owners and it is a condition of accessing publications that users recognise and abide by the legal requirements associated with these rights.

- Users may download and print one copy of any publication from the public portal for the purpose of private study or research.

- You may not further distribute the material or use it for any profit-making activity or commercial gain

- You may freely distribute the URL identifying the publication in the public portal 


\title{
TOWARDS SEMEN QUALITY ASSESSMENT USING NEURAL NETWORKS
}

\author{
Chr. Linneberg, P. Salamon, C. Svarer, and L.K. Hansen \\ CONNECT, Electronics Institute, build. 349 \\ Technical University of Denmark, \\ DK-2800 Lyngby, Denmark \\ email: linne,salamon,csvarer,lkhansen@eileen.ei.dth.dk \\ and \\ J. Meyrowitsch \\ DynaVision, Copenhagen Science Park, SYMBION \\ DK-2100 Copenhagen $\emptyset$, Denmark
}

\begin{abstract}
The paper presents the methodology and results from a neural net based classification of human sperm head morphology. The methodology uses a preprocessing scheme in which invariant Fourier descriptors are lumped into "energy" bands. The resulting networks are pruned using Optimal Brain Damage. Performance comparable to the error rate for human technicians is obtained.
\end{abstract}

\section{INTRODUCTION}

Semen quality assessment is important to fertility studies and standards have been introduced by the World Health Organization[1]. Recent research has demonstrated that semen quality has decreased by $\mathbf{5 0 \%}$ during the past $\mathbf{5 0}$ years in the western world, possibly as result of the increasing exposure to pollutants and changes in diet [4, 5]. The Atlas of Sperm Morphology[2] defines 19 classes of sperm cells based on morphology. The characteristic abnormalities involve shape modifications, including multiple heads and tails. Furthermore, sperm cell motility is also important for definition of abnormality. Since sperm cells show great variety, the morphological classification problem represents a complex task. Furthermore, the appearence of the specimen depends on several factors that are only partially controllable, such as reduced imaging quality, caused by co-fixation of precipitates and presence of non-sperm cells. Hence flexible and robust classification tools are necessary. 
In this communication we analyse a neural net approach to automatic cell shape classification. Neural net learning makes it possible to compensate for the problems of specific imaging devices, and the user is not forced to produce an algorithmic description of the discriminant. Rather the same general software can adapt to a given setting based on examples produced by a skilled technician. Robustness is achieved by the networks' ability to generalize. The prime objective in machine learning is the ability to discriminate appropriately for test cases, i.e., examples that are different from the examples used for training of the neural system. Additional robustness can be obtained by careful preprocessing by which we mean model-based information processing, such as extraction of known salient features.

As a first step towards automatic quality assessment we have developed preprocessing algorithms for locating sperm cells and for extraction of salient features of the "head" and the "tail". In this presentation we will discuss the neural classifier for head shape classification. A convenient preprocessing strategy for shape discrimination has been proposed by $[18,11]$ and is based on Fourier analysis. A polygon in the image plane is isomorphic to a periodic sequence in the complex plane. For reviews and further analysis see also $[11,19,16,17,14,8]$. Fourier descriptors are robust to the number of points used in sampling the shape and readily provide features which are invariant to changes in position, orientation, and starting point $[18,11]$.

Neural networks in conjunction with Fourier descriptors have been applied for shape discrimination previously in [13] and more recently in [8]. The objective of [13] was to recognize tools from a mechanical toolbox. The Fourier coefficients were crudely preprocessed by keeping only a few manually selected amplitudes. The results were promising; good performance was obtained with very few examples using a fully connected feed forward neural net trained by standard Backpropagation. However for sperm cell classification we have found that the inherent variability forces us to invoke optimized classifiers and more sophisticated preprocessing schemes. Our neural classifier approach was first described in [6], and in [8] it was tested on two sets of artificial cell shape data. Here we apply the system to real world data. In the context of cell discrimination the approach involves a new feature: the neural networks used have adaptive architectures. In particular, the networks are pruned to obtain the optimal connectivity. The advantage of the pruned networks is that they perform better on test data, ie. they do not simply memorize the training data but are able to generalize better than fully connected architectures. They are also able to select out the relevant inputs and thereby can mitigate the results of using too many input descriptors. More details on the simulator for the design of application specific architectures can be found in $[7,6]$. Based on statistical theories of generalization, the best generalization is expected from the least complex networks. In our simulator we search for the best network with a pruning scheme based on the Optimal Brain Damage technique of [10]. We first train a large network that can easily implement the training set. Subsequently we compute the saliency of the 
weights of the network and delete a fraction of the weights with the lowest saliency. The network is re-trained and the procedure repeated as long as the pruned network is able to implement the training set. For noisy problems, of course, one would tolerate a certain amount of error on the training set in order not to overfit ${ }^{1}$. Our results indicate that pruning improves performance but, however, should be terminated well before reaching the smallest network that is able to do the training set.

\section{SHAPE PREPROCESSING}

As stated in the introduction, we used Fourier shape descriptors, i.e. the inputs to our neural networks were computed from the Fourier coefficients of the sequence of $(x, y)$ coordinate pairs viewed as complex numbers: $z=x+i y$. The complex representation of a sequence of $\mathrm{N}$ points becomes periodic with the definition: $z_{N+k}=z_{k}$. The Fourier representation has the advantage that the description is rather robust to sampling the actual cell shape with different points and is rather insensitive to the number of points in the sample [3]. It also allows for finding descriptors which are invariant to translation, rotation, and the choice of a starting point in our description of the curve $[18,11]$. The invariances are important because they effectively increase the size of the training set. Using invariant inputs is roughly equivalent to enlarging the training set by adding rotated, renumbered etc. versions of each training sample.

The invariances are achieved by transforming the shape to "standard form". For example, the centroid of the shape can be moved to the origin by setting the zeroth Fourier coefficients equal to zero. The standard form inherent in the popular "elliptic Fourier descriptors" [11] is based on the elliptic approximation for the shape which is obtained by truncating the Fourier series to terms with frequency one. This so-called primary ellipse then serves through its major axis to provide a natural starting point and a natural coordinate system which takes care of rotation and scale. The resulting transformed Fourier coefficients are invariants which provide a complete description of the shape and are called the elliptic Fourier coefficients.

To further reduce the complexity of the input representation, hence the network, we lumped the component by summing the energy in certain frequency bands.

\section{NEURAL CLASSIFIER}

Our simulation tool for design of adaptive neural architectures was described in $[7,6]$. The initial architecture is an ordinary feed-forward network with one hidden layer. The standard output coding scheme for multi-class problems is used, hence, each class is represented by a specific output neuron. The

\footnotetext{
${ }^{1}$ For an implementation of this within time series prediction see [7].
} 


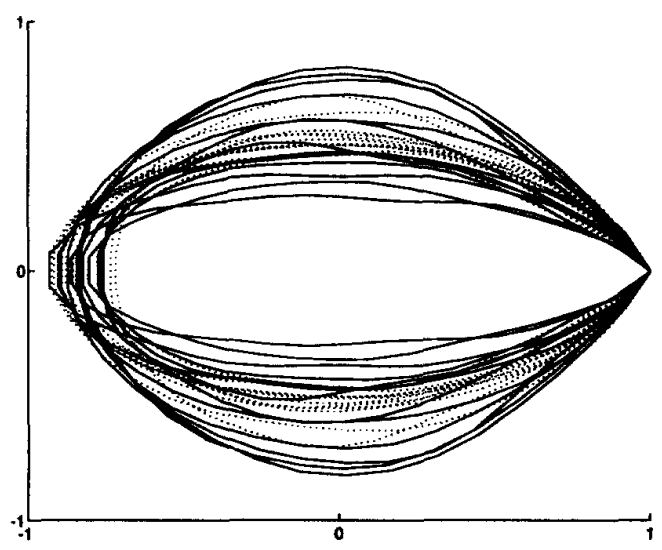

Figure 1: Reconstruction of cells from reduced sets of Fourier coefficient. (Solid: normal cell shapes; dotted: amorphous cell shapes)

activation functions of the hidden neurons are hyperbolic tangents, while the activation functions of the output neurons are linear. The training error is the usual sum of squared errors. The learning algorithm adjusts the weights of the network in order to minimize the error function. For the hidden layer, a second order modification of the back-propagation algorithm with batch learning is used, and, for the output neuron, the matrix inversion algorithm, described in [9] is used.

The algorithm used to adapt the neural network architecture is in brief:

1. Train the fully connected neural network using the learning algorithm.

2. Use the Optimal Brain Damage method to calculate the saliencies of all the weights in the network.

3. Find the weight in the network with the lowest saliency and remove this weight by setting it (permanently) to zero. If a hidden neuron is disconnected from the output neuron the hidden neuron is removed.

4. Retrain the network using the learning algorithm.

\section{THE IMAGE DATABASE}

The image database used in this work consisted of digitized images of fixated and Papanicolau stained semen specimens collected at the Department of Growth and Reproduction, Rigshospitalet Copenhagen. Each image obtained contains a variable number of manually classified cells. The classifications were far from certain and show that the problem includes significant 
overlap between the categories. Six independent laboratory technicians classified the images and on the average the technicians differed from the consensus classification on more than $15 \%$ of the samples. To simplify matters, we have restricted our attention to binary discrimination using two categories: "normal" and "abnormal". The set consisted of 50 cells; 25 from each category. The set was divided into a training set of 13 cells from each category, and a test set of the remaining 24 cells.

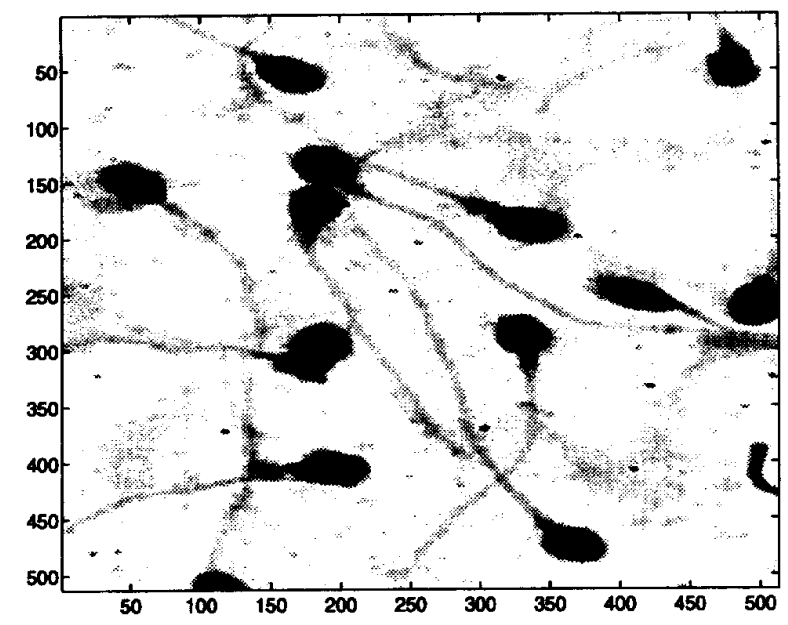

Figure 2: An original grey scale image used for manual labeling of the sperm cells

A

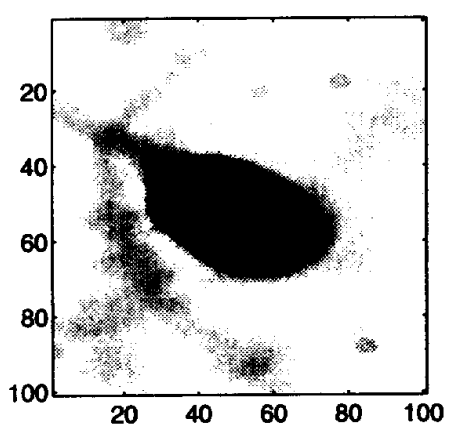

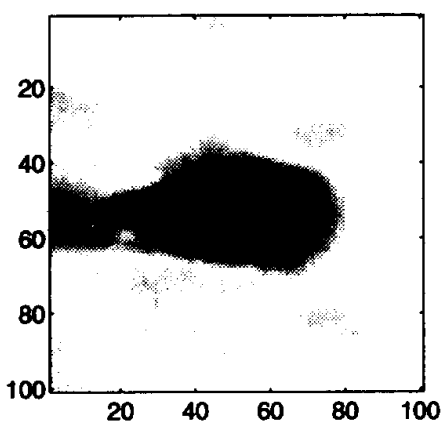

Figure 3: Examples of the two kinds of sperm cells. A) Normal cell shape B) Amorphous shape 


\section{EXPERIMENTAL RESULTS}

The feed forward net was configured with 20 input units. The first 16 input units contained normalized Fourier amplitudes corresponding to 8 lowfrequency pairs. The remaining four inputs represented the accumulated energies of 2 intermediate-frequency bands. The phases of all the Fourier components were discarded along with the amplitudes for the high-frequency components. The network has been initialized with random weights and a simple feed-forward architecture with 6 hidden neurons. It was found that the nets could be pruned significantly and that such pruning improved generalization. The training and test errors during a pruning run are depicted in figure 5. Contrary to previous experience using the pruning strategy $[7,6]$, the network with minimum test error was not the network with the smallest architecture which correctly implements the training set. Furthermore, we suspect that this is generally to be expected for problems with small training sets and significant overlap between categories. We are presently working on a crossvalidation methodology for choosing the best architecture along a pruning sequence.

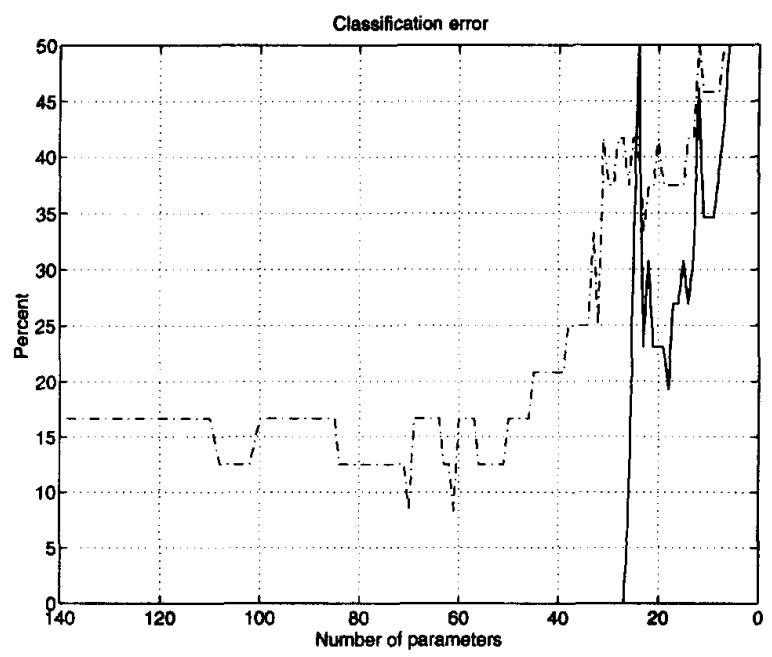

Figure 4: Training (solid line) and test errors (dot-dashed) as pruning progresses. For reference, the test error level of a technician is about $15 \%$.

Inspecting the test errors it is found that pruning can decrease the error by about $25 \%$. 


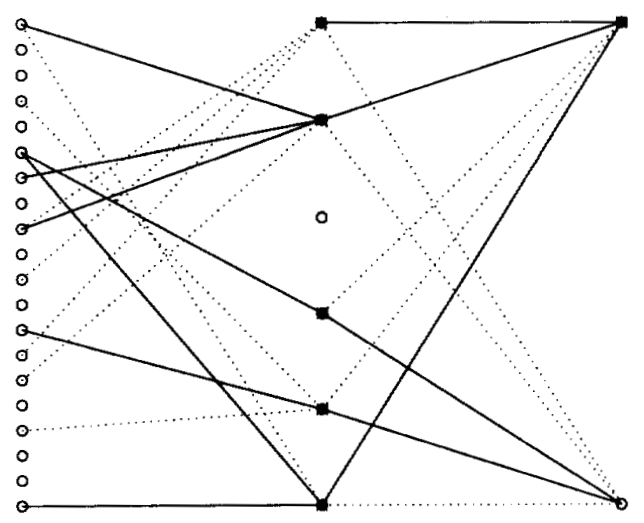

Figure 5: Typical pruned network that can learn the training set (Solid line Positive weight, Dotted line - Negative weight).
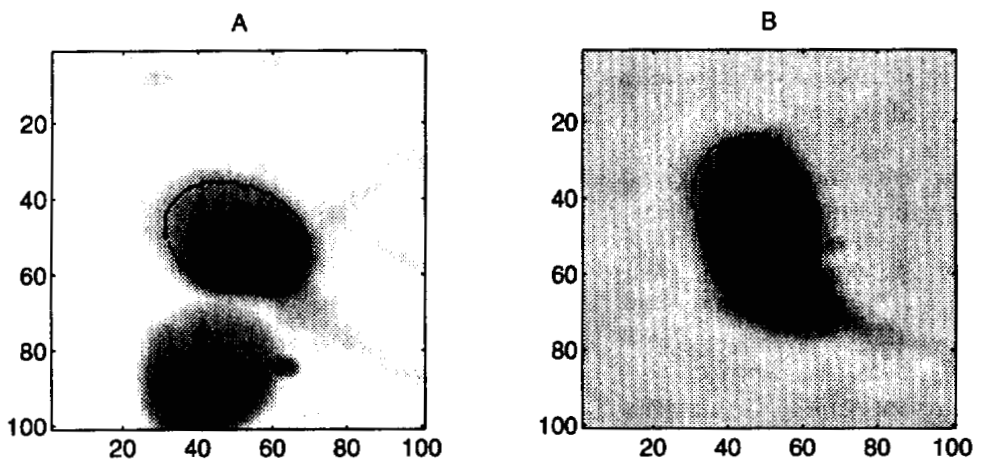

Figure 6: Two cell shapes from the test set that were not learned by the above network. A) normal cell B) amorphous cell. 


\section{CONCLUSION}

It has been shown that neural networks are useful for morphological classification of cell shapes. Application specific architectures can be designed automatically which generalize well to an independent test set. The optimal architectures showed that only a few neurons and connections are necessary. Also it has been shown that preprocessing the cell shape coordinates using the complex Fourier transformation provides a well-suited and compact representation of the data. The optimal networks use less input information than the human eye apparently found necessary. Current work concerns larger databases, multi-class classification, and crossvalidation methodologies for choosing the best network among a pruning sequence.

\section{ACKNOWLEDGMENTS}

This research is supported by the Danish Research Councils for the Natural and Technical Sciences through the Danish Computational Neural Network Center. We thank A. Gewercman and N. Jørgensen (Dept. of Growth and Reproduction, Rigshospitalet, Copenhagen) and Erik Bostofte (Fertilitetsklinikken, Hvidovre Hospital and The Clinic of the medical doctors of Copenhagen) for useful discussions and for providing data for this project.

\section{REFERENCES}

[1] "WHO Laboratory Manual for the Examination of Human Semen and SemenCervical Mucus Interactions" Cambridge University Press, Cambridge 1992.

[2] M.M. Adelman and E.M. Cahill "Atlas of Sperm Morphology" ASCP Press, Chicago (1989).

[3] G.W. Strang "Introduction to Applied Mathematics" Cambridge University Press, Cambridge 1985.

[4] E. Carlsen, A. Gewercman, and N.E. Skakkebæk: "Evidence for decreasing quality of semen during past 50 years" British Medical Journal 305, 609-613 (1992).

[5] R.M. Sharpe and N.E. Skakkebæk: "Are oestrogens involved in falling sperm counts and disorders of the male reproductive tract?" The Lancet $\mathbf{3 4 1}, 1392$ 1395 (1993).

[6] J. Gorodkin, L.K. Hansen, A. Krogh, C. Svarer, and O. Winter: "A Quantitative Study of Pruning by Optimal Brain Damage". Int. Journ. Neural Systems (1993).

[7] C. Svarer, L.K. Hansen, and J. Larsen:

"On Design and Evaluation of Tapped-Delay Neural Network Architectures" The 1993 IEEE Int. Conference on Neural Networks San Francisco. Eds. H.R. Berenji et al., 45-51, (1993) 
[8] C. Svarer, L.K. Hansen B. Wellhouse and P. Salamon: "Classification of Cell Shapes using Designer Networks". In preparation (1994).

[9] Simon A. Barton: "A Matrix Method for Optimizing a Neural Network", Neural Computation 3, 450-459, (1991)

[10] Yann Le Cun, John S. Denker and Sara A. Solla: "Optimal Brain Damage", In Advances in Neural Information Processing Systems II (Denver 1989), ed. D.S. Touretzky, 396-404. San Mateo: Morgan Kaufmann, (1989)

[11] F.P. Kuhl and C.R. Giardina: "Elliptic Fourier Features of a Closed Contour" Computer graphics and image processing 18, 226-258 (1982)

[12] Bill Wellhouse, Department of Mathematical Science, San Diego State University: "Description of cell shape data", Private communication, (1992)

[13] H-H. Wu and R.A. Schowengerdt: "Shape Discrimination Using inveriant Fourier Representation and a neural network classifier" SPIE 1569 Stochastic and Neural Methods in Signal Processing, Image Processing and, Computer Vision 147-154 (1991).

[14] L.M. Kerley and J.R. Knisley: "Complex Vectors and Image Identification" The College Mathematics Journal 24, 166-174 (1993).

[15] J.C. Schon, J.T. Torre-Bueno, and G.B. Stefano: "Microscopic computerassisted analysis of conformational state: reference to neuroimmunology" Advances in Neuroimmonology 1, 252-259 (1991).

[16] G. Diaz, A. Zuccarelli, I. Pelligra, and A. Giani: "Elliptic Fourier Analysis of Cell and Nuclear Shapes" Computers and Biomedical Research 22, 405-414 (1989).

[17] G. Diaz, D. Quacci, and C. Dell'Orbo: "Recognition of cell surface modulation by elliptic Fourier analysis" Computer Methods and Programs in Biomedicine $31,57-62(1990)$.

[18] G.H. Granlund: "Fourier Preprocessing for Hand Print Character Recognition" IEEE Transactions on Computers C-21, 195-201 (1972).

[19] A.W. Partin, J.S. Schoeniger, J.L. Mohler, and D.S. Coffey: "Fourier analysis of cell motility: Correlation of motility with metastatic potential" Proc.Natl.Acad.Sci 86, 1254-1258, (1989). 